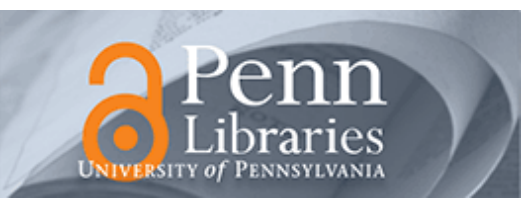

University of Pennsylvania

ScholarlyCommons

\title{
Efficient Generation of Shape-Based Reference Frames for the Corpus Callosum for DTI-based Connectivity Analysis
}

Hui Sun

University of Pennsylvania, sunhui@seas.upenn.edu

Paul A. Yushkevich

University of Pennsylvania, pauly2@grasp.upenn.edu

Hui Zhang

University of Pennsylvania

James C. Gee

University of Pennsylvania, gee@mail.med.upenn.edu

Tony J. Simon

University of California - Davis

Follow this and additional works at: https://repository.upenn.edu/cis_papers

Part of the Engineering Commons

\section{Recommended Citation}

Hui Sun, Paul A. Yushkevich, Hui Zhang, James C. Gee, and Tony J. Simon, "Efficient Generation of ShapeBased Reference Frames for the Corpus Callosum for DTI-based Connectivity Analysis", . June 2006.

Suggested Citation:

Hui Sun; Yushkevich, P.A.; Hui Zhang; Gee, J.C.; Simon, T.J.; , "Efficient Generation of Shape-Based Reference Frames for the Corpus Callosum for DTI-based Connectivity Analysis," Computer Vision and Pattern Recognition Workshop, 2006. CVPRW '06. pp. 87- 87, 17-22 June 2006.

C2006 IEEE. Personal use of this material is permitted. However, permission to reprint/republish this material for advertising or promotional purposes or for creating new collective works for resale or redistribution to servers or lists, or to reuse any copyrighted component of this work in other works must be obtained from the IEEE.

This paper is posted at ScholarlyCommons. https://repository.upenn.edu/cis_papers/441

For more information, please contact repository@pobox.upenn.edu. 


\title{
Efficient Generation of Shape-Based Reference Frames for the Corpus Callosum for DTI-based Connectivity Analysis
}

\author{
Abstract \\ Yushkevich et.al. $[17,18]$ established a PDE-based deformable modeling approach called continuous \\ medial representation (cm-rep), in which the geometric relationship between the medial axis of a 3D \\ object and its boundary is captured. Continuous medial description of an object not only provides useful \\ shape features for object characterization and comparison; it also imposes a shape-based reference \\ frame on the interior of that object. Such a reference frame provides a useful means of representing \\ different instances of an anatomical structure using a common canonical parametrization domain. This \\ paper presents an efficient method to construct continuous medial shape models for 2D objects. A \\ closed form solution for the ordinary differential equation (ODE) is derived via Pythagorean hodograph \\ $\mathrm{PH}$ ) curves. That closed form solution reduces the computation complexity from solving an ODE system \\ to pure algebraic manipulation. Using this method, we generate shape-based reference frames, and \\ demonstrate how they can be applied to the analysis of anatomical connectivity of corpora callosa, \\ obtained by fiber tracking in diffusion tensor magnetic resonance imaging (DTI) in a chromosome \\ 22q11.2 deletion syndrome study.

\section{Disciplines \\ Engineering}

\section{Comments} \\ Suggested Citation: \\ Hui Sun; Yushkevich, P.A.; Hui Zhang; Gee, J.C.; Simon, T.J.; , "Efficient Generation of Shape-Based \\ Reference Frames for the Corpus Callosum for DTI-based Connectivity Analysis," Computer Vision and \\ Pattern Recognition Workshop, 2006. CVPRW '06. pp. 87- 87, 17-22 June 2006. \\ (C2006 IEEE. Personal use of this material is permitted. However, permission to reprint/republish this \\ material for advertising or promotional purposes or for creating new collective works for resale or \\ redistribution to servers or lists, or to reuse any copyrighted component of this work in other works must \\ be obtained from the IEEE.
}




\title{
Efficient Generation of Shape-Based Reference Frames for the Corpus Callosum for DTI-based Connectivity Analysis
}

\author{
Hui Sun, Paul A. Yushkevich, Hui Zhang, James C. Gee \\ Penn Imaging Computing and Science Laboratory, Department of Radiology \\ University of Pennsylvania,Philadelphia,PA,19104,US \\ sunhui@seas.upenn.edu \\ Tony J. Simon \\ Department of Psychiatry and Behavioral Science, M.I.N.D. Institute \\ University of California, Davis, Sacramento, CA, 95817, US
}

\begin{abstract}
Yushkevich et.al. [17, 18] established a PDE-based deformable modeling approach called continuous medial representation (cm-rep), in which the geometric relationship between the medial axis of a 3D object and its boundary is captured. Continuous medial description of an object not only provides useful shape features for object characterization and comparison; it also imposes a shape-based reference frame on the interior of that object. Such a reference frame provides a useful means of representing different instances of an anatomical structure using a common canonical parametrization domain. This paper presents an efficient method to construct continuous medial shape models for $2 D$ objects. A closed form solution for the ordinary differential equation (ODE) is derived via Pythagorean hodograph $(\mathrm{PH})$ curves. That closed form solution reduces the computation complexity from solving an ODE system to pure algebraic manipulation. Using this method, we generate shape-based reference frames, and demonstrate how they can be applied to the analysis of anatomical connectivity of corpora callosa, obtained by fiber tracking in diffusion tensor magnetic resonance imaging (DTI) in a chromosome 22 11.2 deletion syndrome study.
\end{abstract}

\section{Introduction}

With the rapid development of medical imaging field and the wide application of functional magnetic resonance imaging (fMRI) and diffusion tensor magnetic resonance imaging (DTI), medical imaging processing models that can combine shape features and appearance features together are becoming more and more important. The hemodynamic response information provided by fMRI and the diffusiv- ity information from DTI are usually represented as appearance features sampled on a lattice, but a meaningful description of their spatial distribution should take the shape of the anatomical structure into account. Intuitively, "on the tip of structure 1 for instances A, B and C" can be a much more compact and meaningful way to give the spatial location than " $(1.05,2.33,5.1)$ for instance $A,(1.02,2.30,5.01)$ for instance B and $(1.09,2.23,5.2)$ for instance C". That is because the first description is based on the shape analysis result and removes the influence of the shape variation within the population. In other words, we want to obtain a shape-free version of the description. This idea is also well addressed in popular active appearance model (AAM)[4], in which the image is warped into a mean shape image to obtain a shape-free patch of the appearance. The same problem is approached in a different way in continuous medial representation (cm-rep) model, which determines a reference frame tailored to the individual shape of each instance of some anatomical structure and uses that object intrinsicreference frame to parameterize the appearance features. The advantage of cm-rep approach is that first, the reference frame is unique for an object in the sense that it won't depend on the mean shape which may vary according to the data set; second, that reference frame has certain desirable orthogonal properties and its coordinates have meaningful interpretation; and third, that model also gives meaningful shape information like bending and thickness, which can be used for further function-shape co-analysis.

Medial axis is the basic concept in cm-rep. It describes the shape of an object by its skeleton and corresponding thickness field. It represents the shape compactly and provides meaningful shape information. Consequently, it has applications in many fields, such as animation, shape recognition, shape analysis, model based image segmentation and registration. One of it's most appealing advantage is its abil- 


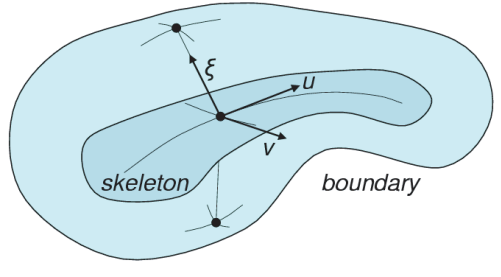

Figure 1. the shape-based reference frame for $\mathrm{cm}$-rep in 3D, coordinates $\{u, v\}$ follow the medial skeleton surface and $\xi$ is orthogonal to the boundary

ity to impose an object intrinsic reference frame. However, although this has been pointed out for a long time, surprisingly, it didn't turn into application until quite recently. One reason lies in the fundamental sensitivity of medial description to object variation and small boundary perturbation. Cm-reps, like their predecessor m-reps developed by Pizer et al. [12], take a generative approach, where templates with a predetermined medial branching topology are fitted to image data. This approach enforces a consistent topology to the medial axis, with the trade off of losing some accuracy in the representation. As a continuous version of m-rep, cm-rep provides a consistent parametric description of the medial axis, which in turn imposes a consistent shape-based reference frame on the interior of the object. In 3D, the first two coordinates follow the medial skeleton surface, and the other goes from the skeleton to the corresponding boundary points, as illustrated in Figure 1; while in 2D, the first coordinate follows the medial skeleton curve. Such reference frame allows us to project the appearance features of different objects into a common canonical coordinate space, in which shape differences between individual objects can be effectively removed.

The method to construct such reference frames in $3 \mathrm{D}$ requires solving a PDE in each fitting iteration. In 3D, that PDE is solved numerically. In 2D, same method involves solving an ODE. In this paper, we present a closed form solution to that ODE based on a class of curves known as Pythagorean hodographs $(\mathrm{PH})[6,5]$, which allow the arc length along the curves to be expressed as polynomial functions. This closed form solution makes the construction of cm-rep in 2D extremely efficient. Actually the system can be constructed purely by manipulation of the coefficients of polynomial curves.

Shape-based reference frame derived from cm-reps have many potential applications in medical imaging, including combined analysis of structure and function in fMRI and combined analysis of shape and connectivity in DTI. As we illustrated in this paper, the $2 \mathrm{D} \mathrm{cm}$-rep method provides a new paradigm for analyzing axon fiber connectivity in the corpus callosum. This paradigm combined fiber tracking, track labeling and normalization to allow anatomical partitioning of the corpus callosum on the basis of in vivo imaging. We illustrate this idea in the context of an ongoing chromosome 22q11.2 deletion syndrome study.

\section{CM-Rep and Shape-Based Reference Frame}

Here we briefly summarize the main framework of cmrep approach and discuss the shape-based reference frame based on literature $[17,18]$, before we move on to investigate the method of cm-rep approach in $2 \mathrm{D}$.

\subsection{Concepts in Medial Geometry}

Medial axis has been studied rigorously in the recent literature $[9,13]$. Here we just give the definitions and facts in medial geometry that are necessary for understanding the cm-rep framework.

1. A n-dimensional object is a bounded set in $\mathbb{R}^{n}$ that is homeomorphic to n-dimensional unit ball and whose boundary is singularity-free.

2. Let $\mathcal{O}$ be a n-dimensional object, a closed ball $\mathcal{B}$ is called a maximal inscribed ball(MIB) of $\mathcal{O}$ if and only if $\mathcal{B} \subseteq \mathcal{O}$ and there does not exist another ball $\mathcal{B}^{\prime}$ such that $\mathcal{B} \subset \mathcal{B}^{\prime} \subseteq \mathcal{O}$.

3. Medial axis of an object $\mathcal{O}$ is the set of points in $\mathbb{R}^{n} \times$ $\mathbb{R}^{+}$formed by the centers and radii of all the MIBs of $\mathcal{O}$. The term skeleton will be used to refer to only the centers of all MIBs in this paper while radial field will be used to refer to the radii of all MIBs.

There are usually 5 types of points in $3 \mathrm{D}$ and 3 types in $2 \mathrm{D}$ that form the medial axis, according to the order and multiplicity of tangency between their MIBs and the boundary of the object[8]. In current cm-rep model, a medial skeleton is assumed to be a single manifold. Under that assumption, a point on medial axis, i.e., the center of certain MIB, can be one of the following two types: if the point (center of the MIB) lies in the interior of the medial skeleton, that MIB will be tangent to $\partial \mathcal{O}$ at two points; if the point (center of the MIB) lies on the boundary of medial skeleton, that MIB will be tangent to $\partial \mathcal{O}$ only at one point. However, since the singly tangent MIBs can be treated as the limit set of the bitangent MIBs, $\mathbf{b}^{+}, \mathbf{b}^{-}$can always be used to denote the two points where the MIB $\mathcal{B}$ is tangent to $\partial \mathcal{O}$. By medial geometry, those boundary points can be obtained by

$$
\begin{aligned}
\mathbf{b}^{ \pm} & =\mathbf{m}+R \overrightarrow{\mathbf{U}}^{ \pm} \\
\overrightarrow{\mathbf{U}}^{ \pm} & =-\nabla_{m} R \pm \sqrt{1-\left\|\nabla_{m} R\right\|^{2}} \overrightarrow{\mathbf{N}}_{m}
\end{aligned}
$$

where $\mathbf{m}$ is the point on medial skeleton (center of MIB), $R$ is the radius, $\overrightarrow{\mathbf{N}}_{m}$ is the unit normal vector of the medial 
skeleton at point $\mathbf{m}, \overrightarrow{\mathbf{U}}^{-}$and $\overrightarrow{\mathbf{U}}^{+}$are unit length vectors orthogonal to $\partial O$ at $\mathbf{b}^{-}$and $\mathbf{b}^{+}$, and $\nabla_{m}$ is the Riemannian gradient on the medial skeleton surface.

According to relation (1), the boundary of an object can be derived from given medial axis, which can be called inverse skeletonization. However, to insure the inverse skeletonization is well-posed, i.e., to insure the given synthetic medial axis can generate a valid object as defined, some constraints must be satisfied. First, for every point on medial axis, inequality constraints

$$
R \geq 0, \quad\left\|\nabla_{m} R\right\| \leq 1
$$

must hold. Then, for the points on the boundary of medial skeleton, the generated boundary points $\mathbf{b}^{+}$and $\mathbf{b}^{-}$must meet there to form a closed object, thus equality constraint

$$
R\left(1-\left\|\nabla_{m} R\right\|^{2}\right)=0
$$

must be satisfied, so that in (1), either $R=0$ or $\overrightarrow{\mathbf{U}}^{-}=\overrightarrow{\mathbf{U}}^{+}$.

Furthermore, to prevent "local" self-intersections of the boundary, a positive Jacobian constraint should be enforced as

$$
J_{\overrightarrow{\mathbf{U}}^{ \pm}}>0
$$

\subsection{PDE-Based CM-Rep Deformable Model}

$\mathrm{Cm}$-rep uses continuous functions to describe the medial axis. Since the medial axis is codimension one to the object it represents, it can be parameterized by $\left(t_{1}, t_{2}\right)$ with $\left(t_{1}, t_{2}\right) \in \Omega$ in $3 \mathrm{D}$; and by $t$ with $t \in[a, b]$ in $2 \mathrm{D}$.

In the cm-rep framework, a medial axis is constructed by first defining its medial skeleton $\mathbf{m}: \Omega \rightarrow \mathbb{R}^{n}$ and an auxiliary scalar field $\rho: \Omega \rightarrow \mathbb{R}$. Its radial field $R$ is then derived from solving a Poisson PDE.

$$
\triangle_{m} \phi=\rho, \text { subject to: }\left\|\nabla_{m} \phi\right\|^{2}=4 \phi \text { on } \partial \Omega
$$

where $\phi=R^{2}$, and $\triangle_{m}$ denotes the Laplace-Beltrami operator on manifold $\mathbf{m}$. The equality constraint (3) is enforced by the boundary conditions of PDE.

Thus, a cm-rep model is defined by the medial skeleton and auxiliary scalar field, which in turn can be defined using a set of basis functions whose coefficients can be adjusted to modify the shape of the cm-rep model. The cm-rep for a particular object is obtained by fitting the deformable cmrep template to the characteristic image in a Bayesian estimation framework. The non-equality constraints (2) and (4) are implemented as prior terms. This procedure for 2D objects will be elaborated in the application section.

\subsection{Shape-Based Reference Frame}

The cm-rep imposes a shape-based reference frame on the cm-rep interior, i.e, the region enclosed by the cm-rep generated boundary. Every point $\left.\mathbf{m}\right|_{\left(t_{1}, t_{2}\right)}$ on the medial skeleton is the center of a MIB, that MIB determines two vectors $\left.R \overrightarrow{\mathbf{U}}^{ \pm}\right|_{\left(t_{1}, t_{2}\right)}$, which are refered to as spokes. They start from the MIB center and end at the MIB tangency points to the boundary (a pair of scaffold vectors coincide if that MIB's center is on the medial skeleton boundary $\partial \Omega$ ). All those vectors form a scaffold and span the whole cm-rep interior. So, for every point enclosed by the cm-rep boundary, its position will be determined if two things are known: which vector pair it belongs to and where it is on that vector pair. Therefore, in $3 \mathrm{D}$, a shape-based coordinate can be defined as $\left(t_{1}, t_{2}, \xi\right)$ with $\left(t_{1}, t_{2}\right) \in \Omega$ and $\xi \in[-1,1]$ such that the Cartesian Coordinate of a point can be obtained by

$$
\mathbf{X}\left(t_{1}, t_{2}, \xi\right)=\mathbf{m}\left(t_{1}, t_{2}\right)+|\xi| R\left(t_{1}, t_{2}\right) \overrightarrow{\mathbf{U}}^{\operatorname{sign}(\xi)}
$$

There are some important properties of that coordinate system.

1. The map of coordinates to points is onto.

2. The map of coordinates to points is one-to-one except a codimension one set of points, that is, those points with $\left(t_{1}, t_{2}\right) \in \partial \Omega$, where the scaffold vector pair superpose. So, for those points, $\left(t_{1}, t_{2}, \xi\right)=\left(t_{1}, t_{2},-\xi\right)$.

3. Since the scaffold vector is orthogonal to the boundary, every point share the same $\left(t_{1}, t_{2}\right)$ with its nearest boundary point.

4. Points with $\left(t_{1}, t_{2}\right) \in \partial \Omega$ are those whose associated nearest boundary points achieve maximal curvature on boundary surface. In other words, the locus of points with coordinates $\left\{t_{1}, t_{2}, \pm 1\right\}$ ( where $\left.\left(t_{1}, t_{2}\right) \in \partial \Omega\right)$ is the ridge of curvature on the boundary.

5. Points on medial skeleton have $\xi=0$ while points on cm-rep boundary have $\xi= \pm 1$. The coordinate $|\xi|$ gives the relative position with respect to the boundary and medial skeleton, and the distance to the boundary is given by $(1-|\xi|) R$.

In $2 \mathrm{D}$, just change the parametrization of the medial skeleton surface $\left(t_{1}, t_{2}\right)$ to parametrization of the medial skeleton curve $(t)$, all properties hold.

\section{Closed Form Solution for ODE in 2D}

In this section, we will describe how the closed form solution in 2D is obtained and how it is used to construct an efficient algorithm.

\subsection{Solution Via Green's Function}

First, let's look at the ODE in 2D. Let $t \in[0,1]$, the Poisson PDE (5) simplifies to the following ODE:

$$
\begin{aligned}
& \frac{d^{2} \phi}{d s^{2}}=\rho(t) ; \\
& \text { subject to: }\left(\frac{d \phi}{d s}\right)^{2}=4 \phi \text { at } t=0,1,
\end{aligned}
$$


where $s$ is the arc length of the medial curve $\alpha(t)=$ $\{x(t), y(t)\}$, i.e, $s(t)=\int_{0}^{t} \sqrt{\left|\alpha^{\prime}(t)\right|} d t$. Note that the boundary conditions are nonlinear, that means if we have two functions $f_{1}$ and $f_{2}$ both satisfy the boundary conditions, $k f_{1}+f_{2}$ usually does not.

Denoting $\sigma(t) \equiv \frac{d s}{d t}$, the above ODE can be expanded using the chain rule

$$
\begin{aligned}
& \left(\frac{\phi^{\prime}(t)}{\sigma(t)}\right)^{\prime}=\rho(t) \sigma(t) ; \\
& \text { subject to: }\left(\phi^{\prime}(t)\right)^{2}=4 \sigma^{2}(t) \phi(t) \text { at } t=0,1,
\end{aligned}
$$

which is a Sturm-Liouville equation with nonlinear boundary conditions. The Green's function $G(t, u)$ for this equation is given by

$$
\left(\frac{G^{\prime}(t, u)}{\sigma(t)}\right)^{\prime}=\delta(t-u)
$$

and the solution is obtained by

$$
\phi(t)=\int_{0}^{1} G(t, u) \rho(u) \sigma(u) d u,
$$

so long as the boundary conditions are satisfied.

Solving equation (9) by integration leads to the following solution

$$
G(t, u)=H(t-u)[s(t)-s(u)]+C_{1}(u) s(t)+C_{2}(u),
$$

where $H$ denotes the Heaviside step function, $C_{1}(u)$ and $C_{2}(u)$ are functions independent of $t$. Since the boundary conditions of $\phi(t)$ are nonlinear, we can not assign a boundary condition to every $G(t, u)$ to determine $C_{1}(u)$ and $C_{2}(u)$. Instead, we substitute the Green's function solutions (11) into equation (10) and use the boundary conditions of (8), which gives us the following relations of functions $C_{1}(u)$ and $C_{2}(u)$

$$
\begin{aligned}
& \int_{0}^{1} C_{1}(u) \rho(u) \sigma(u) d u=D_{1} \\
& \int_{0}^{1} C_{2}(u) \rho(u) \sigma(u) d u=D_{2}
\end{aligned}
$$

where

$$
\begin{aligned}
& D_{1}=\frac{4 L P-4 Q-P^{2}}{2(P-2 L)} \\
& D_{2}=\frac{\left(4 L P-4 Q-P^{2}\right)^{2}}{16(P-2 L)^{2}}
\end{aligned}
$$

Here $L=s(1)$ is the length of the medial curve, $P=$ $\int_{0}^{1} \rho(u) \sigma(u) d u$, and $Q=\int_{0}^{1} s(u) \rho(u) \sigma(u) d u$.

Using the relations (12), we can rewrite the solution of ODE (10) as

$$
\begin{aligned}
\phi(t)= & s(t) \int_{0}^{t} \rho(u) \sigma(u) d u- \\
& \int_{0}^{t} s(u) \rho(u) \sigma(u) d u+D_{1} s(t)+D_{2}
\end{aligned}
$$

Since $s(t)$ is actually an integral of $\sigma(t)$, this solution for $\phi(t)$ actually still involves double integral. That solution doesn't have a closed form for arbitrary regular curves. In order to further simplify it, we take the advantage of Pythagorean hodograph (PH) curves $[6,5,3]$.

\subsection{Closed Form Solution via Pythagorean Hodo- graph Curves}

In this section, we will introduce PH curves to our solution to further simplify it and make it extremely efficient for computation.

$\mathrm{PH}$ curves are polynomial parametric curves $\hat{\alpha}(t)=$ $\{\hat{x}(t), \hat{y}(t)\}$ for which there exists a polynomial $\hat{\sigma}(t)$ such that

$$
\hat{x}^{\prime 2}(t)+\hat{y}^{\prime 2}(t) \equiv \hat{\sigma}^{2}(t)
$$

For PH curves, $\left\{\hat{x}^{\prime}, \hat{y}^{\prime}, \hat{\sigma}\right\}$ form a Pythagorean triple, so that not only $\hat{x}(t)$ and $\hat{y}(t)$, but also the arc length $\hat{s}(t)$ along the curve, can be expressed as a polynomial function.

In [10], it is proved that $\mathrm{PH}$ curves must have the form

$$
\begin{aligned}
\hat{x}^{\prime}(t) & =w(t)\left[p^{2}(t)-q^{2}(t)\right], \\
\hat{y}^{\prime}(t) & =2 w(t) p(t) q(t), \\
\hat{\sigma}(t) & =w(t)\left[p^{2}(t)+q^{2}(t)\right] .
\end{aligned}
$$

where $w(t), p(t)$ and $q(t)$ are polynomial functions.

If we use $\mathrm{PH}$ curve (or piecewise $\mathrm{PH}$ curve) to define $\alpha(t)$, and also define $\rho(t)$ as a (piecewise) polynomial function, then all functions in (14) are (piecewise) polynomial functions or integrals of (piecewise) polynomial functions, so that a closed form of $\phi$ can be obtained with ease. We can also obtain $x(t)$ and $y(t)$ immediately if we are given the boundary condition $(x(0), y(0))$. In practice, we only need to manipulate the coefficients of those polynomial functions, which is extremely fast.

However, if $w(t)$ crosses zero, the given $\{x(t), y(t)\}$ pair would fail to generate a regular curve because $\frac{d s}{d t}$ vanishes there. To avoid that, we can simply set $w(t)=1$. At the same time, since we use piecewise curves, by subdividing the whole curve into enough pieces, we still have enough freedom to deform the curve to fit the anatomical structure.

Therefore, a cm-rep can be completely defined by the coefficients of $p(t), q(t), \rho(t)$ and a translation term $\left(x_{0}, y_{0}\right)$.In current implementation, $p(t), q(t)$ and $\rho(t)$ are approximated by uniform quadratic B-spline functions. Therefore the generated medial curves are piecewise 5th-order polynomial curves, while $\phi(t)$ are piecewise 12th-order polynomial functions, and overall both medial curves and $\phi(t)$ are $C^{2}$.

\section{Application to DTI-based Corpus Callosum Connectivity Map}

In this section, we demonstrate the $2 \mathrm{D}$ cm-rep deformable modeling and shape-based reference frame by applying the method to normalize the DTI-based labeling of the corpora callosa from an ongoing 22q11.2 syndrome study [14]. The DTI-based labeling of corpus callosum is created by labeling each pixel of a 2D midsagittal cross section of the corpus callosum by estimating the most probable cortical to which the pixel is connected by axon fibers. 


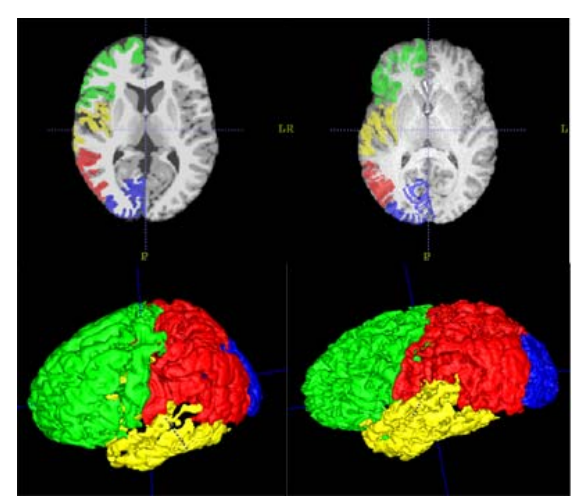

Figure 2. The manually delineated atlas (left) and an example of the atlas warped into the space of a subject's T1-weighted image (right). 2D slices of the cortical labels overlaid with the T1weighted images are shown at the top while $3 \mathrm{D}$ renderings of the cortical labels are shown at the bottom. (Green for frontal lobes, red for parietal lobes, blur for occipital lobes, yellow for temporal lobes)

This labeling scheme leverages the anatomical connectivity of corpus callosum axon fibers to the cortex derived from DTI tractography, thus allows the corpus callosum to be partitioned into subregions on the basis of in vivo imaging, providing a potential alternative to the widely adopted $\mathrm{Wi}$ telson partition, which is based on empirical evidence from post-mortem studies [15]. The normalization of corpus callosum labeling from different subjects to a common canonical parametrization domain allows us to analyze the connectivity pattern of inter-hemispheric fibers through corpus callosum without the influence of statistical confounds due to shape variations.

In the following, we will first describe the generation of DTI-based corpus callosum labeling, then detail the normalization of the labeling results using the deformable cm-rep modeling, finally show the analysis results of the partitionings in the common parametrization domain.

\subsection{Generation of Connectivity-Based Labeling of Corpus Callosum}

Diffusion-weighted and T1-weighted MR scans of 3 typical developing children and 10 children with the 22q11.2 syndrome were acquired on a $3 \mathrm{~T}$ Siemens scanner. For each subject, the diffusion tensor image was reconstructed from its diffusion-weighted scans [2]. The deterministic streamline-based fiber tracking algorithm (FACT)[11] was then applied to the diffusion tensor image to identify white matter fibers inter-connecting the left and right hemispheres of brain through the corpus callosum. We then labeled the cortical regions of the diffusion tensor image by first aligning a manually delineated anatomical atlas to the T1weighted image using a diffeomorphic image registration
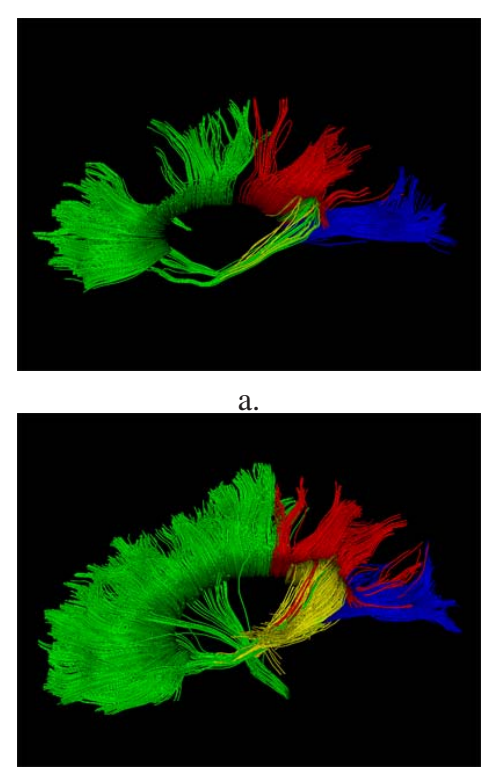

b.

Figure 3. The labeled white matter fibers of the corpus callosum (Green for frontal lobes, red for parietal lobes, blur for occipital lobes, yellow for temporal lobes). Panel a. shows an example of a control child and panel b. shows an example of a child with 22q11.2 syndrome.

algorithm [1] and then placing the warped atlas (in the space of the T1-weighted image) to the space of the diffusion tensor image using the transformation that coregisters the T1weighted image to the diffusion tensor image. The labeling of the anatomical atlas divides the cortex into four regions: frontal, parietal, temporal and occipital, as shown in Figure 2. Each fiber derived from tractography was then assigned the label of the cortical region closest to its endpoints, as shown in Figure 3. Finally, we manually segmented the midsagittal cross-section of the corpus callosum using ITKSNAP [16]. A labeling of the corpus callosum midsagittal based on cortical connectivity was obtained by assigning each pixel the label of the fibers passing through that pixel, as shown in Figure 4. When a pixel had passing fibers with different labels, it was given the label of the fibers with the largest number. Further details can be found in [7].

\subsection{Fitting CM-Rep to Corpus Callosum}

In our current implementation, as we have discussed in section 3 , a cm-rep for a $2 \mathrm{D}$ object is defined by a sequence of control coefficients

$$
\left\{c_{0}^{p}, \cdots, c_{N}^{p}, c_{0}^{q}, \cdots, c_{N}^{q}, c_{0}^{\rho}, \cdots, c_{N}^{\rho}, x_{0}, y_{0}\right\}
$$




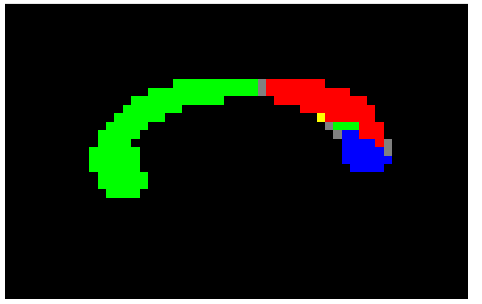

a.

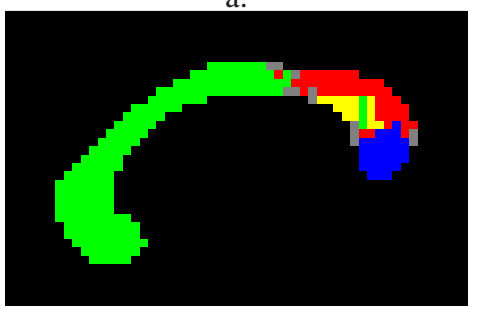

b.

Figure 4. DTI-based color labeled connectivity maps in corpus callosum(Green for frontal lobes, red for parietal lobes, blur for occipital lobes, yellow for temporal lobes).

so that

$$
\begin{aligned}
p(t) & =\sum_{i=0}^{N} c_{i}^{p} B_{i, 2}(t), \\
q(t) & =\sum_{i=0}^{N} c_{i}^{q} B_{i, 2}(t), \\
\rho(t) & =\sum_{i=0}^{N} c_{i}^{\rho} B_{i, 2}(t) .
\end{aligned}
$$

where $B_{i, 2}(t)$ is the uniform quadratic B-spline basis function.

For each corpus callosum instance, we determine the sequence of control coefficients that maximize the posterior probability given the characteristic image of the instance. The posterior probability is factored into a likelihood term and a prior term. The likelihood term measures the match between the cm-rep model and the image. And the prior term can be used to enforce the inequality constraints. In the future, we also intend to build the cm-rep probabilistic atlas and include that in the prior term.

Currently we use $16 \times 3+2$ coefficients in the template. We initialize the fitting by aligning the cm-rep template to the object by a similarity transform. That could be done by a rotation and scaling of vectors $\left(c_{0}^{p}, \cdots, c_{N}^{p}\right)$ and $\left(c_{0}^{q}, \cdots, c_{N}^{q}\right)$ and a translation via changing $\left(x_{0}, y_{0}\right)$. $\rho(t)$ have the good property of invariance under such transforms. Then, Conjugate Gradient Optimization is used to find the optimal set of coefficients. The gradient of the area overlap match can be computed very efficiently by Green's theorem, which converts the double integral over the whole $\mathrm{cm}$-rep interior to a line integral along the $\mathrm{cm}$-rep boundary.

Examples of fitting results are shown in Figure 5. We computed the distance between the fitted cm-rep to the object. The mean distance from cm-rep to object for all 13 instances is $0.55( \pm 0.04)$ pixels, the maximum distance from cm-rep to object is $1.25( \pm 0.14)$ pixels, the mean distance

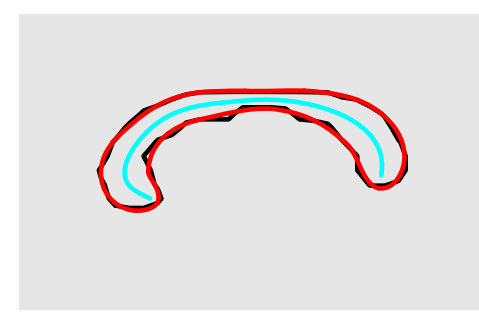

a.

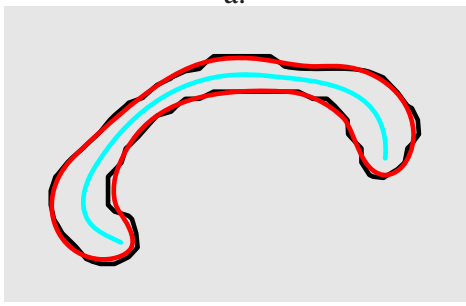

b.

Figure 5. Examples of fitted cm-reps to corpora callosa, the black lines indicate the boundary contour of characteristic image, the red lines are boundary curves of fitted $\mathrm{cm}$-reps, the blue lines are medial skeleton curves.

from object to cm-rep is $0.57( \pm 0.05)$ pixels, and the maximum distance from object to cm-rep is $1.52( \pm 0.36)$ pixels. The representation error of cm-rep is in sub pixel order. The mean Dice similarity coefficient $\left(\frac{2(A \cap B)}{A+B}\right)$, which measures overlap between the binary image and fitted $\mathrm{cm}$ rep for 13 corpora callosa is $91.59( \pm 1.21) \%$, the mean Dice coefficient between the boundary contour image and fitted cm-rep, however, is $93.61( \pm 0.92) \%$. The overlap ratio is underestimated by those big pixels along the boundary. We expect a higher overlap ratio for image with better resolution.

\subsection{Connectivity Map Analysis in Shape-Based Reference Frame}

The natural consistent parametrization for curves is by its arc length, which we can use to establish the correspondence for medial skeleton curves. It would take extra effort for establishing correspondence for medial skeleton surfaces among 3D objects since such "natural consistent" parametrization doesn't exist for surface.

For the fitted cm-rep, let the normalized arc length $\frac{s(t)}{L}$ serve as the new parametrization of the medial skeleton curve and use it as the first coordinate, meantime use $\xi$ as the second coordinate. We then obtained the shape-based reference frame for each instance of corpora callosa. Figure 6 shows the grid lines of the shape-based reference frame imposed by the fitted cm-reps, overlaid on the corpora callosa connectivity maps.

That shape-based reference frame allows us to project connectivity labels into a common canonical coordinate 


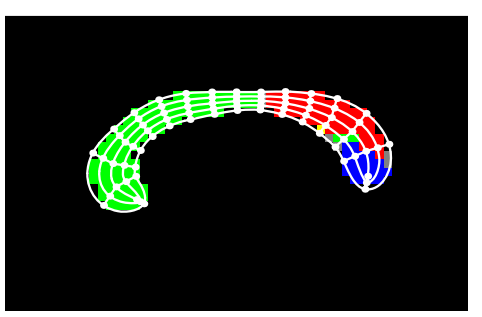

a.

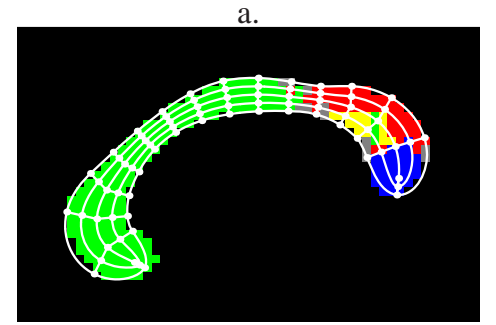

b.

Figure 6. The grid lines of the shape-based reference frame imposed by fitted $\mathrm{cm}$-reps, overlaid on the connectivity maps of corpora callosa. (Green for frontal lobes, red for parietal lobes, blur for occipital lobes, yellow for temporal lobes)

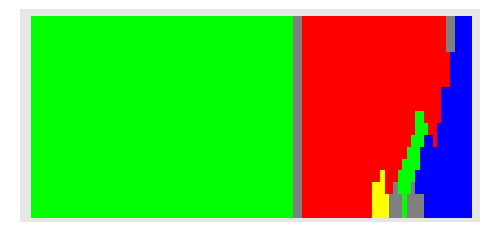

a.

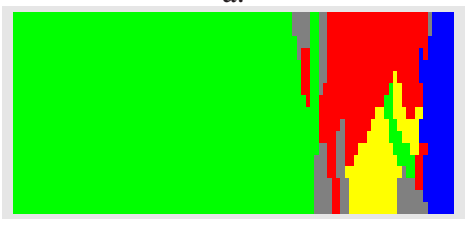

b.

Figure 7. The connectivity maps of corpora callosa in new shapebased reference frame. (Green for frontal lobes, red for parietal lobes, blur for occipital lobes, yellow for temporal lobes)

space, as illustrated in figure 7. In such space, the shape differences between corpora callosa have been effectively removed, which allows us to compare the connectivity map across subjects with ease. As we mentioned before, the map has certain desirable properties: the normal directions to the boundaries are preserved; the boundaries of objects are still mapped to the boundaries in new reference frame; and the medial skeleton curves are mapped to the centerlines. The coordinates in this reference frame have meaningful interpretation too, which is potentially helpful for research.

In this case, since more information lies along the medial skeleton curves, further dimensionality reduction can be achieved by plotting the connectivity density for each

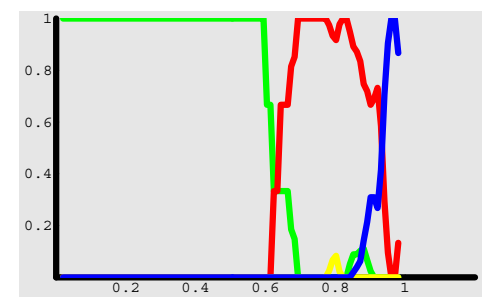

control.

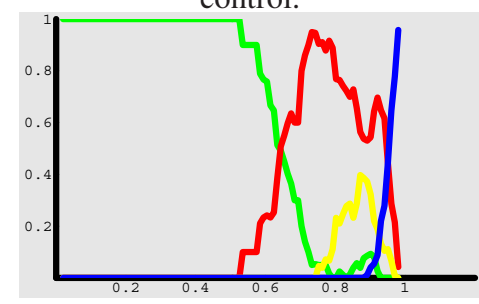

patient.

Figure 8 . The mean connectivity density along medial skeleton for different populations. (Green for frontal lobes, red for parietal lobes, blur for occipital lobes, yellow for temporal lobes)

cortical region against the first coordinate of the canonical space, collapsing values along the second coordinate. Figure 8 shows mean profiles for patients and controls obtained by averaging such $1 \mathrm{D}$ profiles across subjects. It appears that the density of fibers connecting to temporal lobes is greater in patients, however, the relatively small size of the data set precludes us from making statistically significant statements. We expect to be able to detect differences between groups as the number of subjects in our ongoing study increases.

Here we established the correspondence for medial skeleton curves by parameterizing them using normalized arc length, which can be used to compare the connectivity along the medial skeleton curves among subjects. On the other hand, we can choose to establish correspondence by a simple $1 \mathrm{D}$ registration based on the connectivity map, which can be used to compare the length of medial skeleton curves connected to different cortical regions across. The ability to label the corpus callosum according to cortical connectivity in vivo, combined with the ability to analyze resulting maps of labels statistically, may potentially be used to generate a partitioning of the corpus callosum that would be an alternative to the more empirical Witelson partition [15], which was based on post-mortem experiments. We will explore this direction in future research.

\section{Conclusion}

We have developed an efficient method to construct a cm-rep model for 2D objects. This cm-rep model imposes a shape-based reference frame, which can represent different instances of an anatomical structure using a common parametrization domain, in which the shape differences between 
objects are effectively removed. This shape-based reference frame can be useful in the DTI and fMRI study, as we illustrated by a case study on DTI based connectivity analysis in corpus callosum.

\section{References}

[1] B. Avants, P. Schoenemann, and J. Gee. Landmark and intensity-driven lagrangian frame diffeomorphic image registration: Application to structurally and functionally based inter-species comparison. Medical Image Analysis special issue on WBIR 2003, 2005. in press. 5

[2] P. J. Basser, J. Mattiello, and D. L. Bihan. Estimation of the effective self-diffusion tensor from the NMR spin echo. JMR, 103:247-254, 1994. 5

[3] H. I. Choi, C. Y. Han, H. P. Moon, K. H. Roh, and N.-S Wee. Medial axis transform and offset curves by minkowski pythagorean hodograph curves. Computer-Aided Design, 31, 1999. 4

[4] T. F. Cootes, G. J. Edwards, and C. J. Taylor. Active appearance models. IEEE Trans. Pattern Anal. Mach. Intell, 23(6):681-685, 2001. 1

[5] R. T. Farouki and C. A. Neff. Hermite interpolation by Pythagorean hodograph quintics. j-MATH-COMPUT, 64(212):1589-1609, Oct. 1995. 2, 4

[6] R. T. Farouki and T. Sakkalis. Pythagorean hodographs. IBM Journal of Res. and Dev., 34:736-752, 1990. 2, 4

[7] J. C. Gee, H. Zhang, A. Dubb, and et al. Anatomy-based visualizations of diffusion tensor images of brain white matter. In J. Welckert and H. Hagen, editors, Visualization and Image Processing of Tensor Fields. Berlin:Springer, 2005. 5

[8] P. Giblin and B. Kimia. A formal classification of 3D medial axis points and their local geometry. In IEEE Computer Society Conference on Computer Vision and Pattern Recognition, pages 566-573, 2000. 2

[9] P. J. Giblin and B. B. Kimia. On the intrinsic reconstruction of shape from its symmetries. IEEE Transactions on Pattern Analysis and Machine Intelligence, 25(7):895-911, 2003. 2

[10] K. K. Kubota. Pythagorean triples in unique factorization domains. Amer. Math. Monthly, 79:503-505, 1972. 4

[11] S. Mori, B. J. Crain, V. P. Chacko, and P. C. M. van Zijl. Three dimensional tracking of axonal projections in the brain by magnetic resonance imaging. Ann Neurol, 45:265-269, 1999. 5

[12] S. M. Pizer, P. T. Fletcher, S. Joshi, A. Thall, J. Z. Chen, Y. Fridman, D. S. Fritsch, A. G. Gash, J. M. Glotzer, M. R Jiroutek, C. Lu, K. E. Muller, G. Tracton, P. Yushkevich, and E. L. Chaney. Deformable m-reps for 3D medical image segmentation. International Journal of Computer Vision, 55(2):85-106, Nov 2003. 2

[13] S. M. Pizer, K. Siddiqi, G. Székely, J. N. Damon, and S. W. Zucker. Multiscale medial loci and their properties. International Journal of Computer Vision, 55(2-3):155-179, 2003. 2

[14] T. J. Simon, L. Ding, J. P. Bish, D. M. McDonald-McGinn, E. H. Zackai, and J. Gee. Volumetric, connective and morphologic changes in the brains of children with chromosome 22q11.2 deletion syndrome: An integrative study. NeuroImage, 25:169-180, 2005. 4

[15] S. F. Witelson. Hand and sex differences in the isthmus and genu of the human corpus callosum. a postmortem morphological study. Brain, 112:799-835, 1989. 5, 7

[16] P. A. Yushkevich, J. Piven, H. Cody Hazlett, R. Gimpel Smith, S. Ho, J. C. Gee, and G. Gerig. User-guided 3D active contour segmentation of anatomical structures: Significantly improved efficiency and reliability. Neuroimage, 2006. To appear. 5

[17] P. A. Yushkevich, H. Zhang, and J. C. Gee. Parametric medial shape representation in 3-D via the Poisson partial differential equation with non-linear boundary conditions. In G. Christensen and M. Sonka, editors, Information Processing in Medical Imaging, pages 162-173, 2005. 1, 2

[18] P. A. Yushkevich, H. Zhang, and J. C. Gee. Statistical modeling of shape and appearance using the continuous medial representation. In Medical Image Computing and ComputerAssisted Intervention, MICCAI, volume 2, pages 725-732, 2005. 1, 2 